\title{
APLICAÇÃO DA PENEIRA MOLECULAR SBA-15 COMO ADSORVENTE PARA ESPÉCIES POLUENTES DE CÁTIONS METÁLICOS EM SOLUÇÃO AQUOSA.
}

\author{
J. J. RODRIGUES ${ }^{1}$, J. C. MARINHO ${ }^{1}$, V. E. ALMEIDA ${ }^{2}$, G. VERAS $^{2}$ e M. G. F. RODRIGUES ${ }^{1}$ \\ Universidade Federal de Campina Grande ${ }^{1}$, Unidade Acadêmica de Engenharia Química ${ }^{1}$ \\ Universidade estadual da Paraíba ${ }^{2}$, Departamento de Química ${ }^{2}$ \\ E-mail para contato: jocielys@yahoo.com.br/meiry@deq.ufcg.edu.br
}

\begin{abstract}
RESUMO -Neste trabalho sintetizou-se a peneira molecular SBA-15, com o intuito de utilizá-la como adsorvente para espécies poluentes de cátions metálicos em solução aquosa. A SBA-15 foi sintetizada com a seguinte composição molar: 1.0 (fonte de sílica): 0.017 P123: $5.7 \mathrm{HCl}: 193 \mathrm{H}_{2} \mathrm{O}$. A solução de PAR foi preparada a uma concentração de $150 \mathrm{ppm}$. Foram utilizados concentrações de 10, 30 e $50 \mathrm{ppm}$ de chumbo para as adsorções e calibração. Foi preparada uma solução tampão bórax pH 9,00. Os espectros foram registrados utilizando o instrumento UV-Vis/NIR da Perkin Elmer, modelo Lambda 750. Os materiais obtidos foram caracterizados através das técnicas de difração de raios-X (DRX), análise química por (EDX) e Adsorção de Nitrogênio (Método de BET). Através das caracterizações verificou-se a formação da peneira molecular SBA-15. Foi realizada a adsorção variando-se a concentração das soluções iniciais dos cátions metálicos, onde foram observados excelentes valores de remoção de Chumbo, em torno de $60 \%$.
\end{abstract}

\section{INTRODUÇÃO}

Atualmente estimulado pelas pressões exercidas pela legislação ambiental, uma crescente preocupação vem surgindo em relação à preservação do meio ambiente, principal prejudicado pelo continuo aumento de poluentes na atmosfera. A descarga de íons de metais pesados vem prejudicando o ambiente e pondo em risco a saúde humana. Geralmente, esses íons não são biodegradáveis e podem facilmente acumular dentro do corpo humano induzindo a várias doenças (Ulusoy e Akkaya, 2009; Fu e Wang, 2011).

Rostamian e colaboradores (2011) sintetizaram a peneira molecular MCM-41 e utilizaram este material na remoção de metais pesados. O material foi aplicado como adsorvente para os metais pesados $\mathrm{Hg}^{2+} \mathrm{Pb}^{2+} \mathrm{E} \mathrm{Cd}^{2+}$. Os dados de adsorção indicam a seletividade do material para $\mathrm{Hg}^{2+}$ A capacidade de adsorção foi mais alta para $\mathrm{Hg}^{2+}\left(209,6 \mathrm{mg} \mathrm{g}^{-1}\right)$ que para $\mathrm{Pb}^{2+}\left(18,5 \mathrm{mg} \mathrm{g}^{-1}\right) \mathrm{e} \mathrm{Cd}^{2+}$ $\left(13,9 \mathrm{mgg}^{-1}\right)$. 


\section{9 a 22 de outubro de 2014 \\ Florianópolis/SC}

Lee et al., (2011) estudaram a capacidade para a adsorção de íon $\mathrm{Cu}(\mathrm{II})$ de adsorventes. A peneira molecular MCM-41 funcionalizada com aminopropil apresentou a melhor atividade para a adsorção de íon $\mathrm{Cu}(\mathrm{II}), 5,9 \mathrm{mg} \mathrm{g}^{-1}$.

Varios estudos tem sido realizados com o intuito de desenvolver adsorventes altamente eficientes, os materiais mesoporosos ordenados tem apresentado resultados satisfatórios no estudo de remoção de íons de metais pesados (Wu e Zhao, 2011; Rostamian et al., 2011; Lee et al., 2011). As peneiras moleculares são suportes atrativos devido à elevada área superficial e dessa forma são susceptiveis a reações de funcionalização.

A SBA-15 é normalmente sintetizada em meio fortemente ácido, utilizando o tetraetilortosilicato (TEOS) como fonte de sílica e o copolímero tribloco Pluronic PEO-PPO-PEO (P123-Aldrich) como direcionador. Os copolímeros triblocos consistem de um bloco central de polioxipropileno (PPO) e blocos laterais de polioxietileno (PEO) (Zhao, 1998).

A SBA-15 é uma sílica mesoporosa com estrutura hexagonal altamente ordenada possui uma rede hexagonal de mesoporos de aproximadamente $6,0 \mathrm{~nm}$ de diâmetro e elevada área superficial, em torno de $800 \mathrm{~m}^{2} / \mathrm{g}$ (Zhao et al., 1998). Esse material apresenta uma elevada estabilidade térmica e hidrotérmica quando comparado aos materiais pertencentes à família M41S, devido à maior espessura das paredes de seus poros (Zhao et al., 1998).

Neste sentido, este trabalho tem como objetivo a síntese e caracterização da peneira molecular SBA-15 como novo sólido adsorventes, assim como realizar o estudo da capacidade de adsorção de cátions metálicos em solução aquosa. As amostras foram caracterizadas por Difração de Raios X (DRX), Espectrômetro de Raios X por Energia Dispersiva (EDX e Adsorção física de $\mathrm{N}_{2}$ ) e testes de capacidade de adsorção de cátions metálicos em solução aquosa

\section{MATERIAIS E MÉTODOS}

\section{Síntese da peneira molecular SBA-15}

Para a síntese da peneira molecular SBA-15 foram utilizados os seguintes reagentes: Pluronic P123 (poli(óxido de etileno)-poli(óxido de propileno)-poli(óxido de etileno),PEO20PPO70PEO20)) como direcionador, ácido clorídrico 37\%, água destilada, Tetraetilortosilicato (TEOS) como fonte de sílica, baseado no método hidrotérmico proposto por Zhao et al.(1998). Estes reagentes foram misturados em ordem para obter um hidrogel reativo com composição molar: 1,0 FONTE DE SÍLICA(TEOS): 0,017 P123(DIRECIONADOR): 5,7 HCl: $193 \mathrm{H} 2 \mathrm{O}$. A síntese foi realizada da seguinte forma: dissolveu-se o direcionador $\mathrm{P} 123$ em água destilada e $\mathrm{HCl}$, com agitação e aquecimento até $35^{\circ} \mathrm{C}$. Atingida a temperatura, adicionou-se a fonte de sílica. A mistura foi mantida sob agitação e aquecimento a $35^{\circ} \mathrm{C}$ por 24 horas $(\mathrm{pH}=1)$ para obter um gel homogêneo. Depois deste período o gel de síntese foi transferido para autoclave e acondicionado em estufa por 48 horas, previamente aquecida a $100^{\circ} \mathrm{C}(8,9)$. Após esse processo o material obtido foi calcinado, em uma 
mufla, da temperatura ambiente até $550{ }^{\circ} \mathrm{C}$ com uma taxa de aquecimento de $10^{\circ} \mathrm{C} / \mathrm{min}$ permanecendo nesta temperatura por 24 horas.

\section{Realização de Ensaios de Banho Finito (Teste de Adsorção)}

Para a realização dos ensaios de banho finito as amostras de efluentes contendo as emulsões, foram preparadas de acordo com a matriz de planejamento. Onde foram adicionados $0,5 \mathrm{~g}$ de SBA-15 a $50 \mathrm{~mL}$ das emulsões com concentrações de 10,30 e $50 \mathrm{ppm}$ em recipiente, erlenmeyer de $125 \mathrm{~mL}$. Depois de adicionado as emulsões, o conjunto (solução (Chumbo) + SBA-15) foram levados para a agitação mecânica, variando entre $100 \mathrm{rpm}$ durante 3 horas, de forma a garantir o equilíbrio do sistema (CURBELO, 2002).

\section{Determinações de chumbo por espectrofotometria UV-Vis/NIR.}

Para determinação das concentrações de chumbo foi utilizado um aparelho UV-Vis/NIR. A amostra (chumbo) foi misturada com a solução complexante de PAR (4-(2-piridilazo) resorcinol), em concentrações pré-determinadas. Inicialmente, foi verificado o espectro de absorção do PAR e das soluções contendo nitrato de chumbo com solução tampão de bórax (Tetraborato de Sódio) no intervalo de 470 a $650 \mathrm{~nm}$, estabelecendo-se como adequado para as medidas de concentração o comprimento de onda de $547 \mathrm{~nm}$. Por meio de uma curva de calibração, variando de $5 \mathrm{ppm}$ em 5ppm, cada leitura de absorbância foi convertida em concentração de chumbo correspondente.

A solução de PAR foi preparada a uma concentração de $150 \mathrm{ppm}$. Foram utilizados concentrações de 10, 30 e 50 ppm de chumbo para as adsorções e calibração. Foi preparada uma solução tampão bórax pH 9,00. Os espectros foram registrados utilizando o instrumento UV-Vis/NIR da Perkin Elmer, modelo Lambda 750.

\section{Caracterização}

Difração de Raios X (DRX): Os difratogramas de raios-X foram realizados utilizando-se radiação de $\mathrm{K} \alpha$ de $\mathrm{Cu}$, com tensão acelerada $40 \mathrm{KV}$ e corrente de $40 \mathrm{~mA}$, com varredura de $0,5^{\circ}$ a $8^{\circ}$ para $2 \theta$, com velocidade de varredura de $2 \%$ min, realizados em um difratômetro XRD-6000 da Shimadzu.

Análise Química através do Espectrômetro de Raios X por Energia Dispersiva (EDX): A composição química das amostras foi analisada em um espectrômetro EDX-700 Shimadzu.

Adsorção de Nitrogênio (Método de BET): As características texturais dos catalisadores foram avaliadas a partir de isotermas de adsorção e dessorção de nitrogênio a $77 \mathrm{~K}$ obtidas através do equipamento ASAP 2020 da Micromeritics. Para isso $100 \mathrm{mg}$ de cada amostra foi previamente tratada nas as seguintes condições: Taxa de aquecimento: $10^{\circ} \mathrm{C} / \mathrm{min}$, Temperatura: $90^{\circ} \mathrm{C}$, Vácuo setpoint: 10 $\mu \mathrm{mHg}$, Tempo de vácuo: $60 \mathrm{~min}$. Fase de aquecimento: Taxa de aquecimento: $10^{\circ} \mathrm{C} / \mathrm{min} \mathrm{e}$ Temperatura de $350^{\circ} \mathrm{C}$. 


\section{RESULTADOS E DISCUSSÃO}

\section{Difração de Raios X}

Os difratogramas de raios X da peneira molecular SBA-15 estão apresentados na Figura 1.

a)

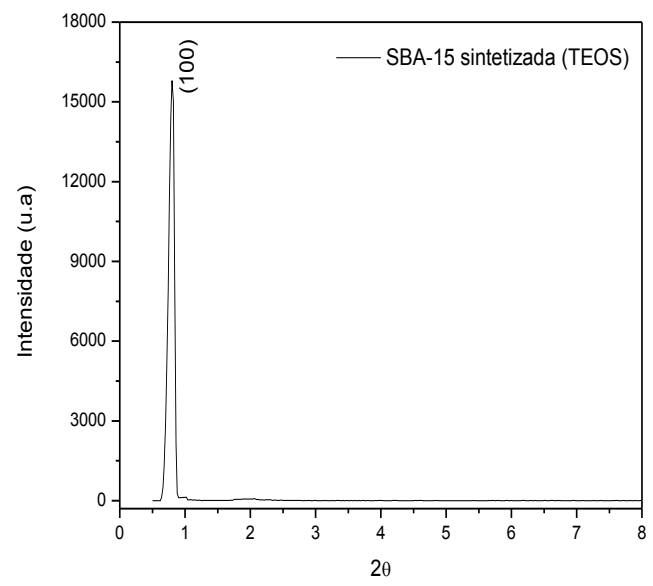

b)

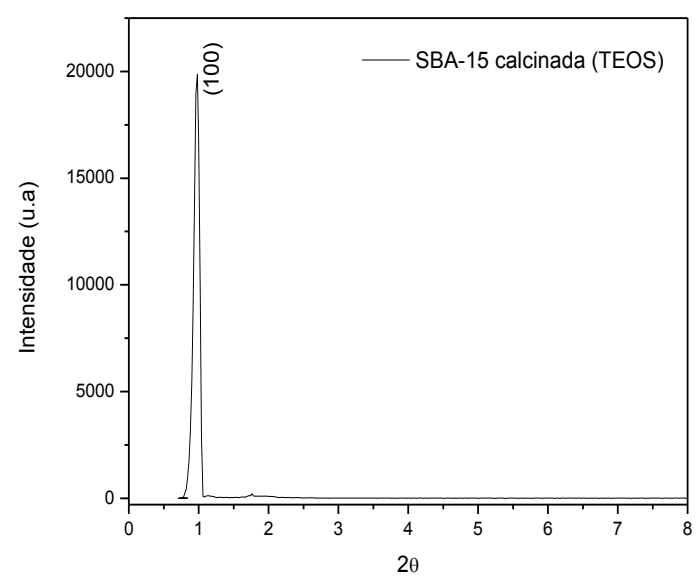

Figura 1 - Difratogramas de Raios X da peneira molecular SBA-15: a) Sintetizada e b) Calcinada.

Os difratogramas de raios $\mathrm{X}$ da peneira molecular SBA são apresentados na Figura 1a (sintetizada) e 1b (calcinada). Através da análise dos difratogramas, é possível identificar o pico principal de difração, característico desse material, referente ao plano cristalino, cujos índices de Miller são (1 0 0), relativo a estrutura hexagonal ordenada da peneira molecular mesoporosa SBA-15 de acordo com a literatura (Zhao et al., 1998; Rodrigues et al., 2013). No processo de síntese, ao dissolver o direcionador Pluronic P123 em água e ácido clorídrico sob agitação e aquecimento brando $\left(35^{\circ} \mathrm{C}\right)$ são formadas as interações copolímero/solvente. Com a adição da fonte de sílica originam-se interações sílica/solvente, após esse processo ocorrem as interações sílica/copolímero. Após o processo de envelhecimento por 24 horas, o gel obtido pode resultar dependendo das condições da solução (temperatura, $\mathrm{pH}$ ) em uma fase siloxana pela condensação das espécies de sílica sobre as micelas direcionadoras do copolímero, e assim, o gel pode ser submetido a um tratamento hidrotérmico formando a peneira molecular SBA-15.

\section{Energia Dispersiva de Raios X}

A Tabela 1 apresenta os resultados da análise química das amostras de SBA-15. 
Tabela 1 - Composição química da peneira molecular SBA-15.

\begin{tabular}{|c|c|c|}
\hline Amostras & $\mathrm{SiO}_{2}(\%)$ & Outros $(\%)$ \\
\hline SBA-15 (sintetizada) & 99,3 & 0,7 \\
\hline SBA-15 (calcinada) & 99,5 & 0,5 \\
\hline
\end{tabular}

De acordo com os resultados de composição química, observa-se que a peneira molecular SBA15 apresenta alto teor de sílica $\left(\mathrm{SiO}_{2}\right)$, isto já era previsto visto que a SBA-15 possui em sua estrutura apenas sílica. As amostras apresentaram quantidades pequenas de impureza, isso diz respeito a contaminações presentes nas amostras, reagentes (Zhao et al. 1998).

\section{Adsorção de Nitrogênio}

Na Figura 2 está exibida a isotermas de adsorção-dessorção de $\mathrm{N}_{2}$ a $-196{ }^{\circ} \mathrm{C}$ referentes à amostra de SBA-15 calcinada.

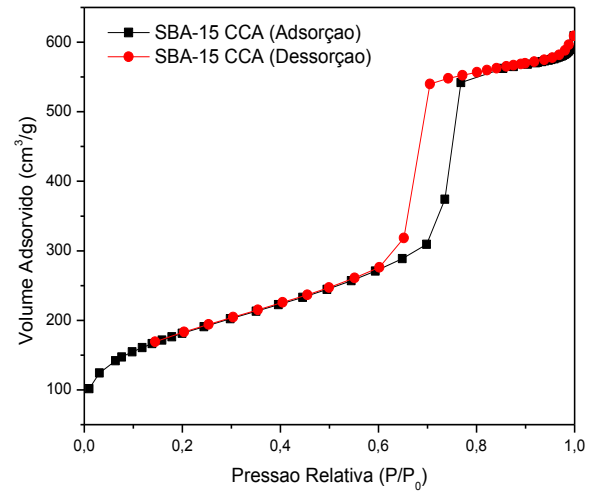

Figura 2 - Isotermas de adsorção-dessorção de $\mathrm{N}_{2}$ a $-196^{\circ} \mathrm{C}$ da peneira molecular SBA-15 calcinada.

Segundo definição da IUPAC, as isotermas obtidas são do tipo IV, característico de materiais mesoporosos. Verifica-se a típica ocorrência de condensação capilar a pressões relativas entre 0,6 e 0,8. É observado o fenômeno de histerese tipo H1, caracterizada por dois ramos de isotermas quase verticais e paralelos durante uma extensa gama de valores do eixo das ordenadas (quantidade adsorvida) (Zhao et al. 1998).

As propriedades texturais da peneira molecular SBA-15 obtidas por adsorção física de $\mathrm{N}_{2}$ são expostas na Tabela 2. 
Tabela 2 - Propriedades texturais da amostra da peneira molecular SBA-15.

\begin{tabular}{|c|c|c|c|c|}
\hline Amostra & $\mathrm{S}_{\mathrm{BET}}\left(\mathrm{m}^{2} / \mathrm{g}\right)$ & $\mathrm{VP}_{\text {total }}\left(\mathrm{cm}^{3} / \mathrm{g}\right)$ & ${ }^{\mathrm{b}} \mathrm{D}_{\mathrm{p}}(\mathrm{nm})$ & $\mathrm{e}(\mathrm{nm})$ \\
\hline SBA-15 & 627 & 0,94 & 6,4 & 6,8 \\
\hline
\end{tabular}

É observado que a área específica, volume total de poros e diâmetro de poros da peneira molecular SBA-15 calcinada $\left(627 \mathrm{~m}^{2} / \mathrm{g}, 0,94 \mathrm{~cm}^{3} / \mathrm{g}\right.$ e $7,4 \mathrm{~nm}$, respectivamente) são característicos de materiais mesoporosos sintetizados em condições similares (Zhao et al. 1998). Através da combinação das técnicas (DRX e BET) pode-se calcular a espessura da parede (Mendes et al. 2006).

\section{Espectrofotometria UV-Vis/NIR}

Através da Tabela 3, podem ser observados os valores das concentrações das soluções de chumbo antes e após remoção, utilizando o SBA-15 como adsorventes.

Tabela 3 - Valores de remoções de chumbo antes e após adsorção.

\begin{tabular}{|c|c|c|c|}
\hline Amostra & $\begin{array}{c}\text { Concentração/ } \mathrm{pH} \\
\text { (antes da } \\
\text { adsorção) }\end{array}$ & $\begin{array}{c}\text { Concentração } \\
\text { (após adsorção) }\end{array}$ & Remoção (\%) \\
\hline 1 & $10 \mathrm{ppm} / \mathrm{pH}=3$ & $7,80 \mathrm{ppm}$ & 22,00 \\
\hline 2 & $50 \mathrm{ppm} / \mathrm{pH}=3$ & $34,74 \mathrm{ppm}$ & 30,50 \\
\hline 3 & $50 \mathrm{ppm} / \mathrm{pH}=5$ & $18,59 \mathrm{ppm}$ & 62,81 \\
\hline 4 & $10 \mathrm{ppm} / \mathrm{pH}=5$ & $5,54 \mathrm{ppm}$ & 44,54 \\
\hline 5 & $30 \mathrm{ppm} / \mathrm{pH}=4$ & $13,26 \mathrm{ppm}$ & 55,78 \\
\hline 6 & $30 \mathrm{ppm} / \mathrm{pH}=4$ & $14,54 \mathrm{ppm}$ & 51,53 \\
\hline 7 & $30 \mathrm{ppm} / \mathrm{pH}=4$ & $13,49 \mathrm{ppm}$ & 55,02 \\
\hline
\end{tabular}

Pode-se verificar através da Tabela 3, foram obtidos valores significativos de adsorções utilizando o SBA-15 como adsorventes. Visto que, os melhores resultados foram obtidos utilizando soluções com pH elevado. Tendo sido obtido a maior remoção de $62,81 \%$ para a amostra com concentração de 50 ppm a pH 5.

\section{CONCLUSÃO}

Através das caracterizações observou-se a formação da peneira molecular SBA-15. As análises de difração de raios $\mathrm{X}$ apresentaram estrutura hexagonal com picos característicos da da peneira molecular SBA-15. As análises de adsorção física de $\mathrm{N}_{2}$ mostram a obtenção de um material com elevada área superficial e volume de poros. 
Os resultados obtidos para remoção de chumbo mostraram melhores resultados no processo com $\mathrm{Ph}$ elevado e o maior percentual de remoção obtido foi $62,80 \%$.

\section{REFERÊNCIAS}

FU, F., WANG, G., Removal of heavy metal íons from wastewaters: A review. Journal of Environmental Management. v. 92, p. 407-418, 2011.

LEE, H. W.; CHO, H.J.; YIM, J.; KIM, J. M.; JEON, J.; SOHN, J. M.; YOO, K.; KIM, S.; PARK, Y., Removal of $\mathrm{Cu}(\mathrm{II})$-ion over amine-functionalized mesoporous silica materials, Journal of Industrial and Engineering Chemistry, v.17, p.504-509, 2011.

MENDES, F M T; PEREZ, C A C; NORONHA, F B; SOUZA, C D D; CESAR, D V; FREUND, H J; SCHMAL, M. Fischer-Tropsch Synthesis on Anchored Co/Nb2O5/A12O3 Catalysts: The Nature of the Surface and the Effect on Chain Growth. Journal of Physical Chemistry, vol. 110, p. 9155-9163, 2006.

ROSTAMIAN, R.; NAJAFI, M.; RAFATI, A. A., Synthesis and characterization of thiolfunctionalized silica nano hollow sphereas a novel adsorbent for removal of poisonous heavy metal ions from water: Kinetics, isotherms and error analysis, Chemical Engineering Journal, v.171, p.1004-1011, 2011.

ULUSOY, U; AKKAYA, R., Adsorptive features of polyacrylamide-apatite composite for $\mathrm{Pb}^{2+}, \mathrm{UO}^{2}$ ${ }^{2+}$ and $\mathrm{Th}^{4+}$, Journal of Hazardous Materials, v.163, p.98-108, 2009.

WU, Z.; ZHAO, D., Ordered mesoporous materials as adsorbents, Chemical Communications, v.47, p.3332-3338, 2011.

ZHAO, E., FENG, J., HUO, Q, FENG, J..G.H., CHMELKA, B.F., STUCKY, G.D. Nonionic triblock copolymer and oligameric surfactant syntheses of highly ordered hydrothermally stable, mesoporous sílica structures. J. Am. Chem. Soc. v.120, p. 6024-6036, 1998.

RODRIGUES, J. J.; FERNANDES, F.A.N.; RODRIGUES, M. G. F. Study of Co/SBA-15 catalysts prepared by microwave and conventional heating methods and application in Fischer-Tropsch synthesis. Appl. Catal. A. v.468, p.32-37, 2013. 